\title{
Modified valsalva versus standard valsalva for cardioversion of supraventricular tachycardia: systematic review and meta-analysis
}

\author{
Ahmed S. Abdulhamid ${ }^{1,3^{*}}$, Fahad Almehmadi ${ }^{1,2}$, Abdullah A. Ghaddaf ${ }^{1,3}$, Mohammed S. Alomari ${ }^{1,3}$, \\ Amin Zagzoog ${ }^{1,2}$ and Atif Al-Qubbany ${ }^{1,2}$
}

\begin{abstract}
Background: Supraventricular tachycardia (SVT) is a major cause of emergency room visits where vagal maneuver is used as first-line therapy. The valsalva maneuver (VM) is proven to be safe and, to some extent, effective in terminating SVT episodes. We aimed to compare the standard VM (SVM) to the modified valsalva maneuver (MVM). We hypothesized that MVM is more effective in terminating SVT episodes and reducing the time spent in the emergency department.

Methods: In this systematic review and meta-analysis, we searched Medline/PubMed, Ovid, Web of Science, and Cochrane Central Register of Controlled trials. We included only randomized controlled trials (RCTs) that compared the modified valsalva to the standard valsalva maneuver in treating SVT. Our main outcome was the termination of SVT within 1 min.

Results: Four articles met the eligibility criteria of our review. Sinus rhythm was achieved 2.5 times more in the MVM group compared to the SVM group (risk ratio $(\mathrm{RR})=2.54, \mathrm{Cl} 1.98-3.24, \mathrm{P}<0.001$ ) and thus lowered the need of intravenous SVT termination medication without any significant increase in adverse events or time spent in the emergency department.
\end{abstract}

Conclusion: Our review found MVM to be more effective than the SVM in terminating SVT. This should encourage broader adoption of the MVM as a first-line vagal maneuver in subjects presenting with SVT in the emergency room.

Keywords: Supraventricular tachycardia, Valsalva maneuver, Trendelenburg position

\section{Background}

Vagal maneuvers are recommended as a first-line modality for terminating supraventricular tachycardia (SVT) in the emergency department (ED) with the standard valsalva maneuver (SVM) being most commonly used [1]. Despite the wide adoption of the SVM in adults presenting with SVT, the efficacy as a sole method in restoring

\footnotetext{
*Correspondence: Abdulhamid018@ksau-hs.edu.sa

${ }^{1}$ College of Medicine, King Saud Bin Abdulaziz University for Health

Sciences, Jeddah, Saudi Arabia

Full list of author information is available at the end of the article
}

sinus rhythm in such patients is less than $25 \%$ [2, 3]. SVM is described as a sustained expiration with a closed glottis for 15-20 s while maintaining a semi-recumbent body position throughout and shortly after. A change in the body position immediately after straining release, in the form of assuming a flat body position along with a $15 \mathrm{~s}$ passive leg raise (known as the modified valsalva maneuver (MVM)), showed higher efficacy $(>40 \%)$ in terminating SVT in ED [2].

(c) The Author(s) 2021, corrected publication 2021. This article is licensed under a Creative Commons Attribution 4.0 International License, which permits use, sharing, adaptation, distribution and reproduction in any medium or format, as long as you give appropriate credit to the original author(s) and the source, provide a link to the Creative Commons licence, and indicate if changes were made. The images or other third party material in this article are included in the article's Creative Commons licence, unless indicated otherwise in a credit line to the material. If material is not included in the article's Creative Commons licence and your intended use is not permitted by statutory regulation or exceeds the permitted use, you will need to obtain permission directly from the copyright holder. To view a copy of this licence, visit http://creativecommons.org/licenses/by/4.0/. 
A large randomized controlled trial (RCT) of 428 participants with SVT randomly assigned to SVM and MVM showed a significantly higher rate of return to sinus rhythm through MVM [2]. Three further RCTs have been published without being pooled in a meta-analysis [3-5].

Given the introduction of 787 participants, we aimed to perform a systematic review and meta-analysis comparing the efficacy SVM and MVM in the termination of SVT in ED with respect to sinus rhythm restoration within $1 \mathrm{~min}$, the need for emergency antiarrhythmic treatment, and time in spent in ED.

\section{Methods}

\section{Search strategy and selection criteria}

This systematic review and meta-analysis included RCTs where there are head-to-head comparisons of SVM and MVM for treating adult patients who presented with SVT in ED. For a study to be included, it must report at least one of the following outcomes: termination of SVT within $1 \mathrm{~min}$, the emergency needs for intravenous aborting antiarrhythmic treatment, adverse event, or time spent in the ED.

We conducted this systematic review and meta-analysis according to a prespecified protocol following the systematic review and meta-analysis PRISMA checklist [6]. We searched Medline/PubMed, Evidence-Based Medicine review databases via Ovid, Web of Science, and Cochrane Central Register of Controlled trials with no restrictions on date or language. We also extended our search into unpublished clinical trial registries such as ClinicalTrials.gov, MetaRegister of Controlled Trials, Australian New Zealand Clinical Trials Registry, and UMIN Clinical Trials Registry. We also searched the bibliographic references of the included RCTs for relevant studies that might be missed in the original search. The last search was done mid-June 2020 .

Two independent investigators in duplicate (AA, MA, and AG) intensively reviewed articles titles and abstracts for the articles that met our prespecified eligibility criteria. Then the same two investigators read the full text of each included studies and extracted all the data needed for our proposed issue using a predefined data collection sheet. Any disagreement was resolved by discussion or the decision of a third reviewer. Data extracted into the spreadsheet included study design, patients' demographic features (mean age and gender), vital signs of the patients at presentation, and the number of participants in each group. The desired outcomes were also extracted including the efficacy data in the form of SVT termination within $1 \mathrm{~min}$, the need of antiarrhythmic abortive therapy, time spent in the ED, and safety data in the form of adverse event rates.

\section{Data synthesis and analysis}

The main outcome of interest was the termination of SVT within $1 \mathrm{~min}$, while secondary outcomes were the need for emergency abortive antiarrhythmic medication, reported adverse events related to either of the valsalva maneuvers, and time spent in the ED. Pooled effect estimates of all events of interest were presented as risk ratio (RR) except for the time spent in ED, which is presented as standardized mean difference (SMD). In two of the included articles, the authors reported the result of time in the ED as a median and interquartile range, so we converted these results into a mean and standard deviation using the calculator provided by Wan et al. [7].

Forest plots were constructed using Comprehensive Meta-Analysis software version 3 using the randomeffects model, and heterogeneity was assessed using $I^{2}$ test. The Cochrane bias risk assessment tool [8] was used for quality assessment where funnel plot and egger's tests were used to depict publication bias. A two-sided $P$ value of $<0.05$ was considered to be significant.

\section{Results}

The literature search yielded 875 articles. After removing the duplicated and excluding non-eligible articles, four were RCTs included in this review (Fig. 1) [2-5]. The included RCTs were published between 2015 and 2019.

\section{Risk of bias assessment}

Two studies (Appelboam et al. and Çorbacıoğlu et al.) had low bias risk, while the other two had high risk. (Table 1) $[2,5]$. The result of the funnel plot for the primary outcome, return to sinus rhythm, is symmetrical, and egger's test $P$ value was not significant $(P=\mathrm{ns})$.

\section{Trial characteristics}

The four included RCTs enrolled 787 participants with SVT in which SVM was used in 394 participants and MVM used in the remaining 393, all included in the meta-analysis. In total, 223 participants were male (107 in SVM and 116 in MVM) and 326 were females (168 in SVM arms and 158 in the MVM ones). The mean age of the participants ranged between 44.3 and 61 years. The presenting heart rate of clinical tachycardia ranged from 167 beats per minute (BPM) to 180 BPM in SVM and 172 BPM to 180 BPM in MVM (Table 2).

\section{Return of sinus rhythm}

Return of sinus rhythm in 1 min was reported by all studies [2-5]. MVM was more effective in restoring sinus rhythm than SVM $(\mathrm{RR}=2.54$, CI 1.98-3.24, $P<0.001$; $\left.I^{2}=0 \%\right)($ Fig. 2). 


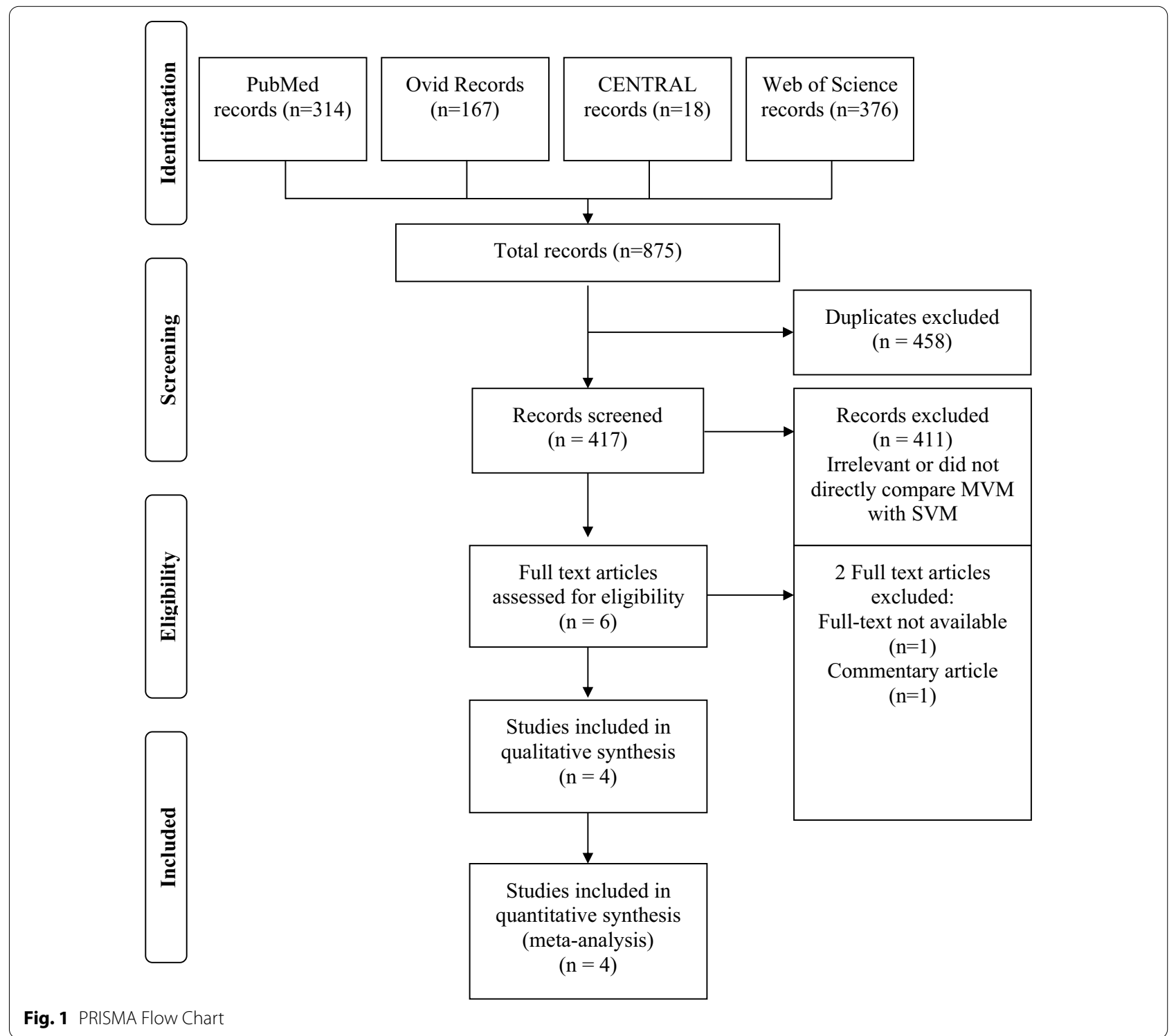

Table 1 Risk of bias assessment

\begin{tabular}{|c|c|c|c|c|c|c|}
\hline Study & Randomization & $\begin{array}{l}\text { Deviations from the } \\
\text { intended intervention }\end{array}$ & $\begin{array}{l}\text { Missing } \\
\text { outcomes data }\end{array}$ & $\begin{array}{l}\text { Measurement of the } \\
\text { outcome }\end{array}$ & $\begin{array}{l}\text { Selection of the } \\
\text { reported results }\end{array}$ & $\begin{array}{l}\text { Overall } \\
\text { risk of } \\
\text { bias }\end{array}$ \\
\hline Appelboam et al. [2] & $\ominus$ & $\ominus$ & $\ominus$ & $\ominus$ & $\ominus$ & $\ominus$ \\
\hline Chen et al. (2019) & ? & $\ominus$ & $\ominus$ & $\ominus$ & ? & $\oplus$ \\
\hline Ceylan et al. [3] & $\oplus$ & $\ominus$ & $\ominus$ & $\ominus$ & $\ominus$ & $\oplus$ \\
\hline Çorbacıoğlu (2017) [5] & $\ominus$ & $\ominus$ & $\theta$ & $\theta$ & $\ominus$ & $\theta$ \\
\hline
\end{tabular}

$\oplus:$ High Risk, $\ominus$ : Low Risk, ??: Some Concerns

\section{Use of abortive antiarrhythmic medications}

The use of abortive antiarrhythmic medications was reported by three studies and was found to be less common in participants randomized to $\mathrm{MVM}(\mathrm{RR}=0.68, \mathrm{CI}$ 0.61-0.76, $P<0.001 ; I^{2}=0 \%$ ) $[2,4,5]$ (Fig. 3).

\section{Adverse events}

Adverse events were reported by three trials [2, 4, 5]. The rate of adverse events did not differ between the two maneuvers $\left(\mathrm{RR}=1.53\right.$, CI $\left.0.73-3.23, P=\mathrm{ns} ; I^{2}=0 \%\right)$ (Fig. 4). 


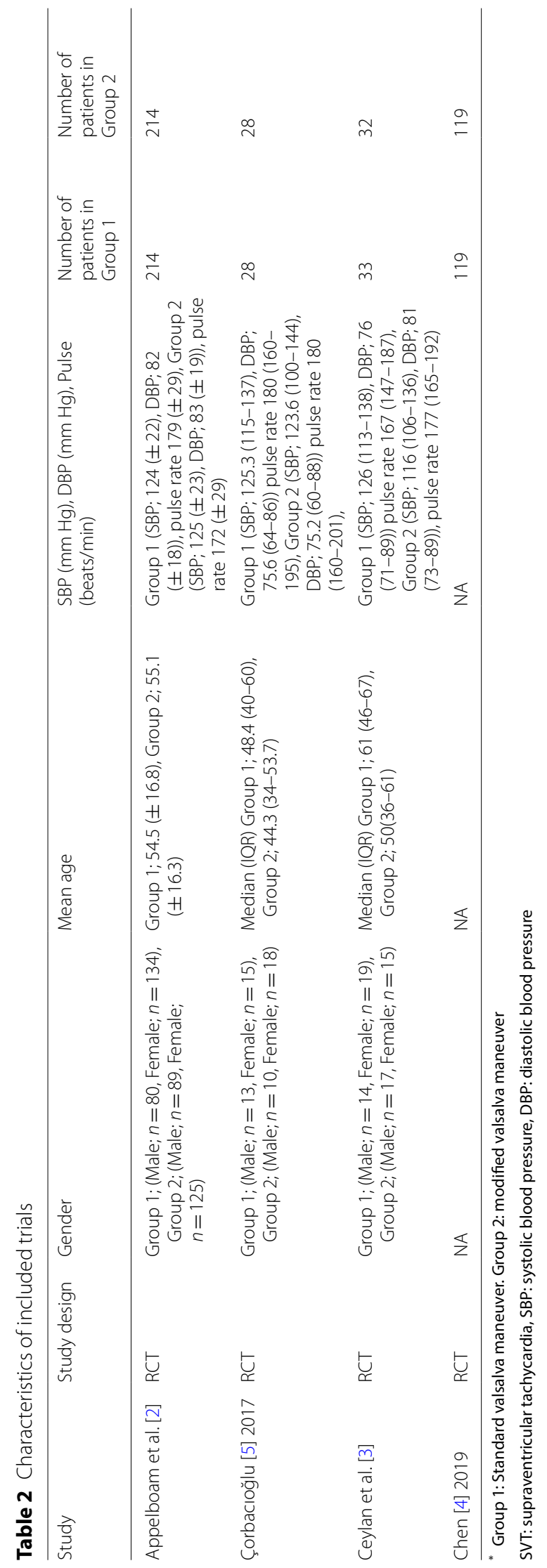




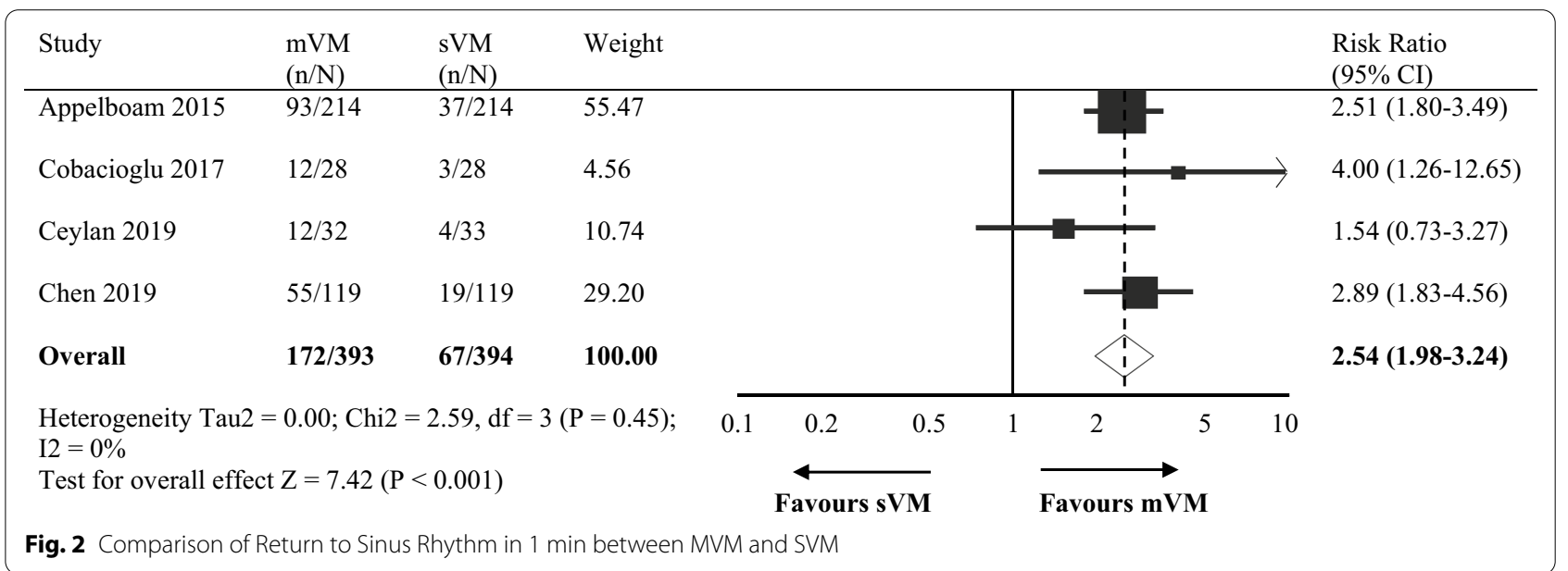

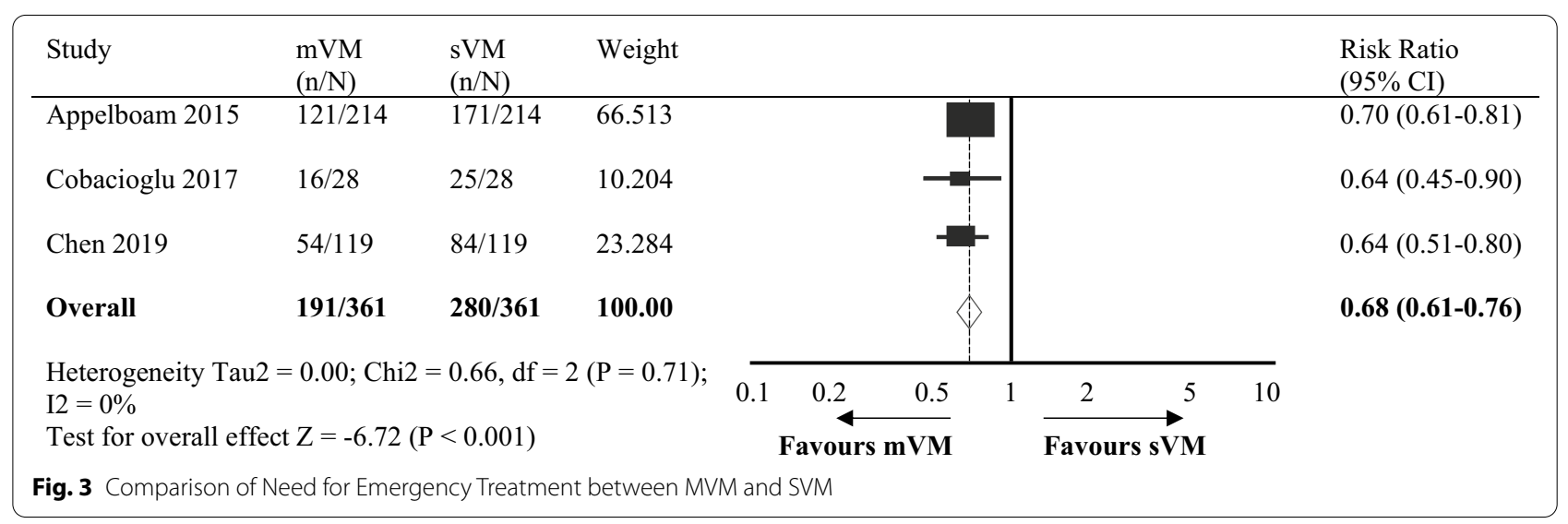

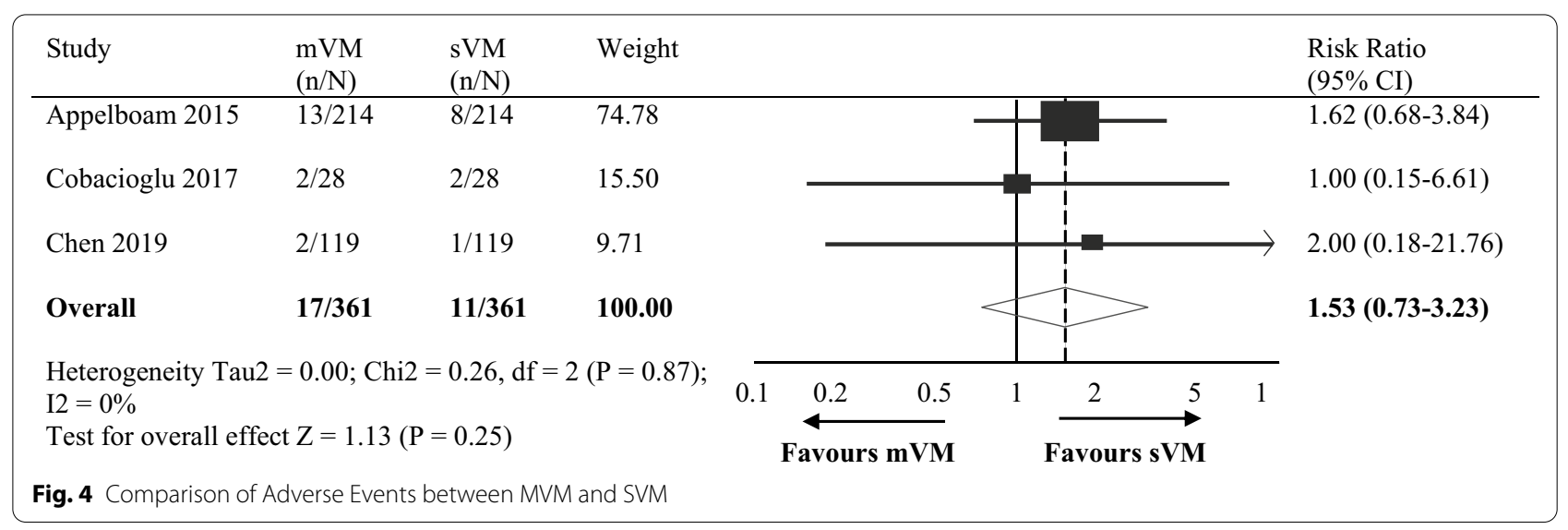

\section{Time in ED}

Time in ED was reported by three studies [2, 4, 5]. No significant difference was found between MVM and SVM $\left(\mathrm{SMD}=0.002, \mathrm{CI}-0.14-0.14, P=0.97 ; I^{2}=0 \%\right)$ (Fig. 5).

\section{Discussion}

In this systematic review and meta-analysis, we provide a high level of evidence results that are consistent with most studies done on the same issue. Our result showed 


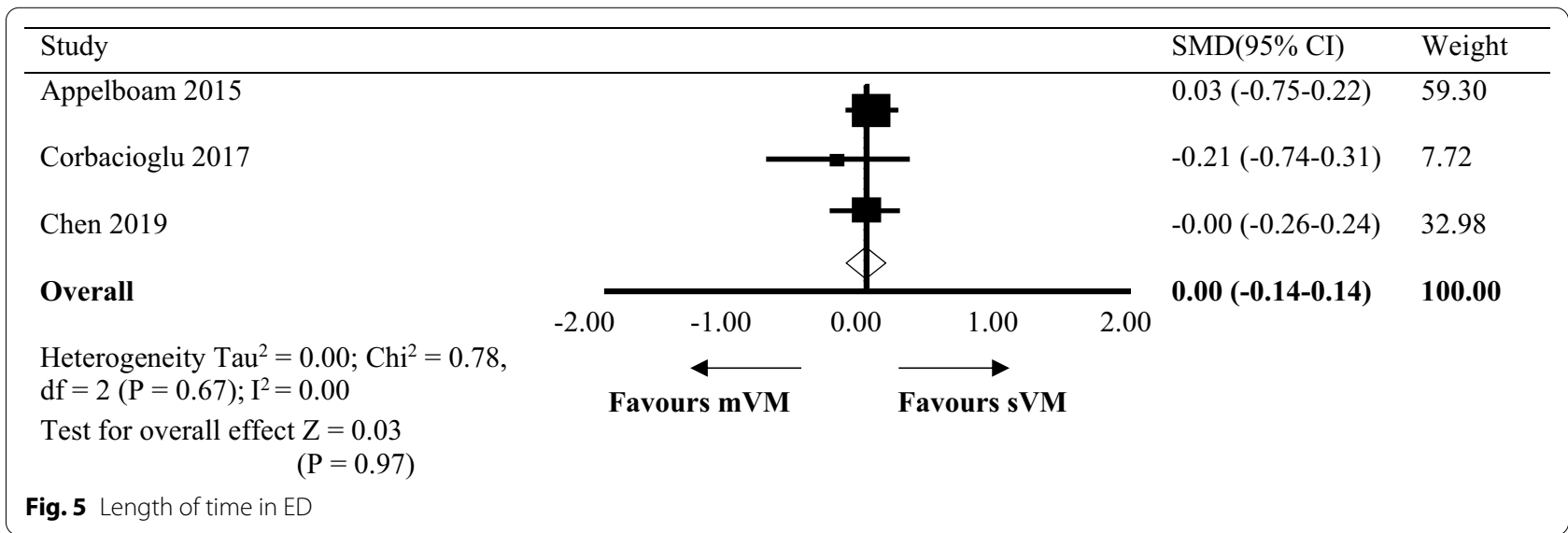

that using the MVM in patients with SVT decreased the need for any emergency treatment by terminating the SVT with simple, cost-free, and well-tolerated intervention.

The efficacy of the valsalva maneuver is dependent on generating high intrathoracic pressure and stimulating baroreceptors in the carotid body and aortic arch leading to an increase in the vagal tone and venous return [9]. The subjectivity of straining efforts might explain the variation (in the efficacy) of SVM efficacy [10, 11]. This is a limitation in delivering this mode of therapy as it is heavily dependent on the instructions provided to the patients by the healthcare workers (i.e., emergency response personnel and physicians). This can explain the inconsistency in performing the valsalva maneuvers as it has been reported that only $9.6 \%$ of ED physicians would give specific instructions to their patient on the position and the duration, according to Taylor et al. [12]. Patients with recurrent SVT can be taught on how to perform MVM by blowing into a $10-\mathrm{ml}$ syringe, which can result in the same intrathoracic pressure (equivalent to $40 \mathrm{mmHg}$ of generated pressure) as an aneroid manometer yet widely available [9]. Addressing this limitation will lead to faster termination of SVT, reducing ED visits when the vagal maneuver is self-administered, shorter time in ED, lower need of administering IV medication, hospital admission, and cardioversion.

The passive leg rise suggested by Appelbaum et al. study will maximize the venous return which will increase the preload resulting in an increase in the cardiac output causing higher vagal tone response. Appelbaum et al. were the first systematically reported in a systematic method the merit of MVM. In RCT involving 428 patients, MVM was more effective in aborting SVT episodes when compared to SVM (43\% vs. $17 \%$ ) [2]. Following Appelbaum et al., Çorbacioğlu et al., Ceylan et al., and Chen et al. all reported a better efficacy of
MVM when compared to SVM ( $43 \%$ vs. $11 \%$; $38 \%$ vs. $12 \%$; and $46 \%$ vs. $16 \%$, respectively) [2-5]. The description of MVM in each study was the same with minor changes. One modification in Chen et al. was the angle of $90^{\circ}$ in raising the legs, which might produce a slightly higher success rate compared to the $45^{\circ}$ described by other studies. This can be explained by the result presented in Chen et al. study and by the physiology of the maneuver and its effect on the baroreceptors [13]. Similar success rates were also reported in a published abstract by Youssef et al. (MVM 47\% vs. SVM 20\%) that we did not include in our meta-analysis due to lack of access to the full manuscript [14]. A case report is done by Appelboum et al. on a patient with a recurrent SVT over 30 occasions in 13 years. MVM was effective as a cardioversion in this patient when both adenosine and verapamil failed to terminate the recurring SVT [15]. A previous systematic review done by Wheeler suggested that the MVM increased the rate of termination of SVT termination in adult patients in the ER and proposed that making a standardization in valsalva maneuver can increase the effectiveness of vagal maneuvers [16]. Also, a study done by Mohammad et al. reported the overall rate success of MVM as $47.3 \%$ (44/93 patients); however, this study did not compare MVM and SVM, and for this reason, was not included our meta-analysis [17]. In our analysis, we found that adopting MVM leads to 2.5-fold increase in the rate of sinus rhythm and a $32 \%$ reduction in the risk of requiring of abortive antiarrhythmic medication in ED.

Carotid sinus massage is another modality commonly reported with limitations; its efficacy is not better than SVM [3], but the risk of stroke risk was a major limitation, especially in older patients $[18,19]$. Appelbaum et al. reported minor adverse events with VM, including transient hypotension, nausea, and musculoskeletal pain, and did not find passive leg rise leads to be associated with more adverse events [2]. 
It is plausible to think that with the rapid elimination of SVT early in the encounter, we might reduce time in the ED, need for admission, and overall cost of care. Time spent in the ED was not different between the tested VMs in our analysis; both maneuvers take little or no time to perform. Also, the lack of standardized criteria for admission of SVT patients will limit the merit of a meta-analysis in answering such a question. A dedicated RCT with pre-determined admission criteria and a cost analysis plan will support the wide adoption of MVM as a first-line modality in patients presenting with SVT.

\section{Limitations}

There are some limitations of our review. Our sample size was relatively small for a meta-analysis, but the results of all of the studies were concordant in favor of MVM. Also, we were unable to perform a subgroup analysis due to a lack of baseline data, such as different age groups and different pressure-generating methods. Our results apply to adult patients only with no pediatric data. However, it is still possible to believe that MVM is still useful in older pediatric patients who can properly follow instructions properly and Walker et al. mention the use of MVM in children with success [10].

\section{Conclusion}

Modified valsalva maneuver is more effective than the standard maneuver in terminating SVT without increasing adverse events or time spent in the emergency department. Further RCTs are warranted to confirm the efficacy and safety of the MVM.

\section{Abbreviations}

MVM: Modified valsalva maneuver; SVM: Standard valsalva maneuver; SVT: Supraventricular tachycardia; ED: Emergency department.

\section{Acknowledgement}

Not applicable.

\section{Authors' contributions}

ASA contributed to research idea, design of the study, data extraction, statistical analysis and interpretation, risk of bias assessment, writing the first draft, and writing and editing the final manuscript. FA, MD contributed to statistical analysis interpretation, editing the first draft, and writing and editing the final manuscript. AAG and MSA contributed to design of the study, data extraction, statistical analysis and interpretation, risk of bias assessment, writing and editing the final manuscript. AZ, MD, and AA, MD contributed to statistical analysis interpretation and writing and editing the final manuscript.

\section{Funding}

Not applicable.

\section{Availability of data and materials}

The analyzed data in this study are available in the following published articles: (1) Appelboam et al. [2], (2) Çorbacıoğlu et al. [5], (3) Ceylan et al. [3], (4) Chen et al. [4].

\section{Ethics approval and consent to participate}

Appelboam et al. [2] were approved by the South West-Exeter; Research Ethics; Ceylan et al. [3] were approved by Marmara University Pendik Research and Training Hospital Ethics Committee; Chen et al. (2019) were approved by the Institutional Review Board of Jiangsu Province People's Hospital of Xuzhou branch [4]; Çorbacıoğlu et al. [5] were approved by their local ethics committee (Kecioren Training and Research Hospital, Department of Emergency Medicine, Ankara, Turkey).

\section{Consent for publication}

Not applicable.

\section{Competing interests}

We declare no conflicts of interest to disclose.

\section{Author details}

${ }^{1}$ College of Medicine, King Saud Bin Abdulaziz University for Health Sciences, Jeddah, Saudi Arabia. ${ }^{2}$ King Faisal Cardiac Center, Ministry of National Guard Health Affairs, King Abdullah International Medical Research Center, Jeddah, Saudi Arabia. ${ }^{3}$ King Abdullah International Medical Research Center, Jeddah, Saudi Arabia.

Received: 17 November 2020 Accepted: 11 January 2021

Published online: 12 February 2021

\section{References}

1. Brugada J, Katritsis DG, Arbelo E, et al. 2019 ESC Guidelines for the management of patients with supraventricular tachycardia. The Task Force for the management of patients with supraventricular tachycardia of the European Society of Cardiology (ESC). Eur Heart J. 2020;41(5):655-720.

2. Appelboam A, Reuben A, Mann C, et al. Postural modification to the standard valsalva manoeuvre for emergency treatment of supraventricular tachycardias (REVERT): a randomised controlled trial. Lancet. 2015;386(10005):1747-53

3. Ceylan E, Ozpolat C, Onur O, Akoglu H, Denizbasi A. Initial and sustained response effects of 3 vagal maneuvers in supraventricular tachycardia: a randomised. Clinical Trial J Emerg Med. 2019;57(3):299-305.

4. Chen C, Tam TK, Sun S, et al. A multicenter randomised controlled trial of a modified Valsalva maneuver for cardioversion of supraventricular tachycardias. Am J Emerg Med. 2020;38(6):1077-81.

5. Çorbacıoğlu SK, Akıncı E, Çevik Y, et al. Comparing the success rates of standard and modified Valsalva maneuvers to terminate PSVT: a randomised controlled trial. Am J Emerg Med. 2017;35(11):1662-5.

6. Liberati A, Altman DG, Tetzlaff J, et al. The PRISMA statement for reporting systematic reviews and meta-analyses of studies that evaluate health care interventions: explanation and elaboration. Ann Intern Med. 2009; 151(4): W-65-W-94

7. Wan X, Wang W, Liu J, Tong T. Estimating the sample mean and standard deviation from the sample size, median, range and/or interquartile range. BMC Med Res Methodol. 2014;14(1):135.

8. Sterne JAC, Savović J, Page MJ, et al. RoB 2: a revised tool for assessing risk of bias in randomised trials. BMJ. 2019;366:14898.

9. Smith $\mathrm{G}$, Boyle MJ. The $10 \mathrm{~mL}$ syringe is useful in generating the recommended standard of $40 \mathrm{mmHg}$ intrathoracic pressure for the Valsalva manoeuvre. Emerg Med Australas. 2009;21(6):449-54.

10. Walker S, Cutting P. Impact of a modified valsalva manoeuvre in the termination of paroxysmal supraventricular tachycardia. Emerg Med J. 2010;27(4):287-91.

11. Smith G, Morgans A, Boyle M. Use of the valsalva manoeuvre in the prehospital setting: a review of the literature. Emerg Med J. 2009;26(1):8-10.

12. Taylor DM, Wong LF. Incorrect instruction in the use of the valsalva manoeuvre for paroxysmal supra-ventricular tachycardia is common. Emerg Med Australas. 2004;16(4):284-7.

13. Junqueira Jr. LF. Teaching cardiac autonomic function dynamics employing the valsalva (Valsalva-Weber) maneuver. Adv Physiol Educ. 2008;32(1):100-6.

14. Youssef A. OMNGAMEA. Evaluation of modified Valsalva maneuver in treatment of supraventricular tachycardia among adult patients presenting to ER. EHRA 2019. 
15. Appelboam A, Gagg J, Reuben A. Modified Valsalva manoeuvre to treat recurrent supraventricular tachycardia: description of the technique and its successful use in a patient with a previous near fatal complication of DC cardioversion. BMJ Case Rep. 2014. https://doi.org/10.1136/ bcr-2013-202699.

16. Wheeler J. Modified Valsalva maneuver vs. standard Valsalva maneuver on emergency department patients presenting with supraventricular tachycardia. School of Physician Assistant Studies; 2016. p. 587.

17. Mohammad A, Saeed M, Migliore F. Effectiveness of the modified Valsalva maneuver in the emergency management of supraventricular tachycardia. Med J Babylon. 2019;16(2):104-7.

18. van den Brink RB, de Lange FJ. Carotid sinus massage is not a benign intervention. Ned Tijdschr Geneeskd. 2017;161:D1312.
19. van Munster CE, van Ballegoij WJ, Schroeder-Tanka JM, van den BergVos RM. A severe stroke following carotid sinus massage. Ned Tijdschr Geneeskd. 2017:161:D826.

\section{Publisher's Note}

Springer Nature remains neutral with regard to jurisdictional claims in published maps and institutional affiliations.
Ready to submit your research? Choose BMC and benefit from:

- fast, convenient online submission

- thorough peer review by experienced researchers in your field

- rapid publication on acceptance

- support for research data, including large and complex data types

- gold Open Access which fosters wider collaboration and increased citations

- maximum visibility for your research: over $100 \mathrm{M}$ website views per year

At BMC, research is always in progress.

Learn more biomedcentral.com/submissions 